\section{Nanoparticles for lupus}

\section{By Lauren Martz, Staff Writer}

A Yale University group has designed a nanoparticle that selectively delivered an immunosuppressant to immune cells and improved survival in mouse models of lupus. ${ }^{1}$ The nanoparticle delivery platform has been exclusively licensed to Immunova LLC, which is selecting an antiinflammatory compound to take into the clinic.

Lupus is an autoimmune disease characterized by excessive production of proinflammatory cytokines and autoantibodies that cause damage to multiple tissues, including the kidneys, CNS, joints and skin.

Marketed drugs for the disease have side effects including GI toxicities and increased risk of infection from immunosuppression. Thus, a continuing challenge is figuring out how to target and suppress only the immune cells that trigger autoimmunity.

The Yale group, led by Tarek Fahmy, hypothesized that a nanoparticle drug delivery system could selectively deliver immunosuppressants to autoreactive immune cells. Prior work by a University of Virginia team showed that nanoparticles traveled to the sites of renal tissue inflammation in a lupus mouse model. ${ }^{2}$

"We are proposing that the current challenge in autoimmune diseases is not exclusively limited to drug discovery but in essence is a problem of directed drug delivery to pathogenic immune cells," Fahmy told SciBX.

Fahmy, who is associate professor of biomedical and chemical engineering and of immunobiology at Yale, decided to use a nanoparticle delivery platform to administer the small molecule mycophenolic acid (MPA). Roche markets the MPA prodrug CellCept mycophenolate mofetil to prevent organ transplant rejection. CellCept is in Phase III testing for lupus and is used off label for the indication.

The nanoparticle delivery platform consisted of liposomes and biodegradable polymers that were stable and could encapsulate a range of small molecules.

In a mouse model for systemic lupus erythematosus (SLE), the MPAloaded nanoparticles extended survival to 50-51 weeks compared with 38 weeks for saline control, whereas free MPA did not extend survival at the equivalent dose or at a 16-fold higher dose. Treatment also delayed onset of proteinuria and decreased glomerular damage.

Importantly, the nanoparticles did not reduce body weight or cause hepatic or renal toxicities.

Finally, Fahmy and colleagues showed that dendritic cells took up the nanoparticles and decreased both production of proinflammatory cytokines and $\mathrm{T}$ cell activation. Those findings suggested that the nanoparticles suppress the antigen-presenting and proinflammatory functions of dendritic cells.

The findings were published in The Journal of Clinical Investigation.

"Our next steps are focused on the choice of drug candidates to be encapsulated for lupus clinical translation and then initiating safety trials in humans," said Fahmy, while also continuing development of the MPAloaded nanoparticles.

Fahmy's team also is considering other autoimmune indications for the nanoparticle technology and is working with the Human and Translational Immunology Program at Yale to explore using the platform to treat type 1 diabetes.

\section{Getting optimal}

Meanwhile, Immunova has exclusively licensed the platform. The company is optimizing the nanoparticle content and methods of production, while Fahmy and his team are further characterizing the nanoparticle, potential drug candidates and indications under a sponsored research agreement with the company.

Immunova declined to provide a timeline for when nanoparticle-based therapeutics might reach the clinic.

"The next step to consider is the safety of the nanoparticle for use in humans," said Keith Elkon, professor of medicine and head of the Division of Rheumatology at the University of Washington. "The current concern is about medium- and long-term effects of their administration."

Thus, Elkon wanted to see long-term safety studies in animals. He said nanoparticles have been highly reactive with a number of human cell types, including neurons, depending on the type and composition of the nanoparticles. Resulting problems could include carcinogenesis and CNS toxicity due to the nanoparticle crossing the blood brain barrier.

Michael Look, a postdoctoral fellow in Fahmy's lab at Yale, pointed out that although some types of nanomaterials do cause problems that make them unsuitable for use in humans, the team formulated its nanogels "with this concern in mind and chose materials that are biocompatible and have a robust track record of being safe."

The nanoparticle also may carry the risk of immunosuppression. "Dendritic cells are needed for normal immune function, and dampening their antigen-presenting function long term would require consideration of how it affects patients' abilities to fight infection," added Elkon.

According to Look, the team has two reasons to suspect that risk of infection should not necessarily increase with the nanoparticles.

First, they found that total white blood cell numbers remained normal following very high-dose treatment, which is in contrast with conventional methods of immunosuppression, for which depletion of white blood counts is often associated with opportunistic infection. Second, the mice were still able to generate functional immune responses after immunization. "These observations lead us to believe that a patient could still mount an immune response to an infection even with therapy. Though, of course, we'd still have to check to see if this is true in human trials," said Look.

Fahmy said Yale has filed a patent application covering nanoparticle composition and methods of manufacture and use.

Martz, L. SciBX 6(11); doi:10.1038/scibx.2013.256

Published online March 21, 2013 


\section{ANALYSIS}

\section{REFERENCES}

1. Look, M. et al. J. Clin. Invest.; published online March 1, 2013; doi:10.1172/JCl65907

Contact: Tarek M. Fahmy, Yale University, New Haven, Conn. e-mail: tarek.fahmy@yale.edu

2. Scindia, Y. et al. Arthritis Rheum. 58, 3884-3891 (2008)
COMPANIES AND INSTITUTIONS MENTIONED

Immunova LLC, New Haven, Conn.

Roche (SIX:ROG; OTCQX:RHHBY), Basel, Switzerland

University of Virginia, Charlottesville, Va.

University of Washington, Seattle, Wash.

Yale University, New Haven, Conn. 